\title{
Direct Imaging of Localized Anisotropic Acoustic-Phonon Dynamics in $\mathrm{MoS}_{2}$
}

Yichao Zhang ${ }^{1}$ and David J. Flannigan ${ }^{1 *}$

1. Department of Chemical Engineering and Materials Science, University of Minnesota, Minneapolis, MN 55455, USA

* Corresponding author: flan0076@umn.edu

The large elastic strains that can be sustained by van der Waals bonded layered materials (e.g., TMDs) cause significant modification of the electronic band structure through modulation of bond distances and crystal symmetries. This manifests as measureable variations in the electronic and optical properties $[1,2]$. In addition to static (i.e., time-invariant) strain, ultrafast transient strains can be induced in such materials via femtosecond (fs) photoexcitation. For example, in-plane atomic motions and out-of-plane interlayer coupling in $\mathrm{MoS}_{2}$ have been studied with ultrafast reciprocal-space electron and X-ray diffraction $[3,4]$. Similarly, bright-field imaging in ultrafast electron microscopy (UEM) has been used to study the nucleation, propagation, and relaxation of coherent elastic strain waves in a number of TMDs $\left(\mathrm{MoS}_{2}, \mathrm{WSe}_{2}, \mathrm{TaS}_{2}\right)$ arising from the release of impulsive local strain mediated by defects [5-7].

Here, we report the direct imaging with UEM of photoexcited localized anisotropic coherent acousticphonon dynamics in a single, freestanding, multilayer $\mathrm{MoS}_{2}$ flake. Combining picosecond temporal resolution and nanometer spatial resolution in bright-field imaging, we observe and quantify both the inplane nucleation and propagation of acoustic phonons and an interlayer low-frequency breathing mode spatially isolated and localized within two regions of different crystal thickness and along two distinct zone axis directions. Transient structural information induced via fs photoexcitation was extracted by monitoring Bragg diffraction-contrast motion within discrete, nanoscale regions. Propagating and stationary coherent contrast motion occurring within differently-oriented crystal regions occurs via excitation of both intralayer and interlayer phonon modes. The distinct dynamics are resolved within a single field of view and along a single incident electron wavevector. Isolation of the strongly-scattering coherent dynamics reveal a response delay of a few ps for the intralayer mode, indicating a temporal coupling to the low-frequency, interlayer dynamics. Further, the sensitivity of the measurement is such that a rapid dephasing of the breathing mode relative to an adjacent nanoscale crystal region is observed, indicative of a difference of one to three $\mathrm{MoS}_{2}$ layers and a lag in transmission of the phonon between the crystal boundaries that emerges within a few oscillation periods. These results provide new insights into the microscopic nature of intra and interlayer acoustic-phonon dynamics and mode coupling, especially with respect to the dramatic influence of nanoscale morphology on photoinduced strain [8].

[1] T Shen, AV Penumatcha and J Appenzeller, ACS Nano 10 (2016), p. 4712.

[2] D Jariwala et al., ACS Nano 8 (2014), p. 1102.

[3] EM Mannebach et al., Nano Lett. 15 (2015), p. 6889.

[4] EM Mannebach et al., Nano Lett. 17 (2017), p. 7761.

[5] AJ McKenna, JK Eliason and DJ Flannigan, Nano Lett. 17 (2017), p. 3952.

[6] DR Cremons, DA Plemmons and DJ Flannigan, Nat. Commun. 7 (2016), p. 11230.

[7] DR Cremons, DA Plemmons and DJ Flannigan, Struct. Dyn. 4 (2017), p. 044019.

[8] This material is based upon work supported by the National Science Foundation under Grant No. DMR-1654318. Partial support was provided by the Arnold and Mabel Beckman Foundation in the form of a Beckman Young Investigator Award. 

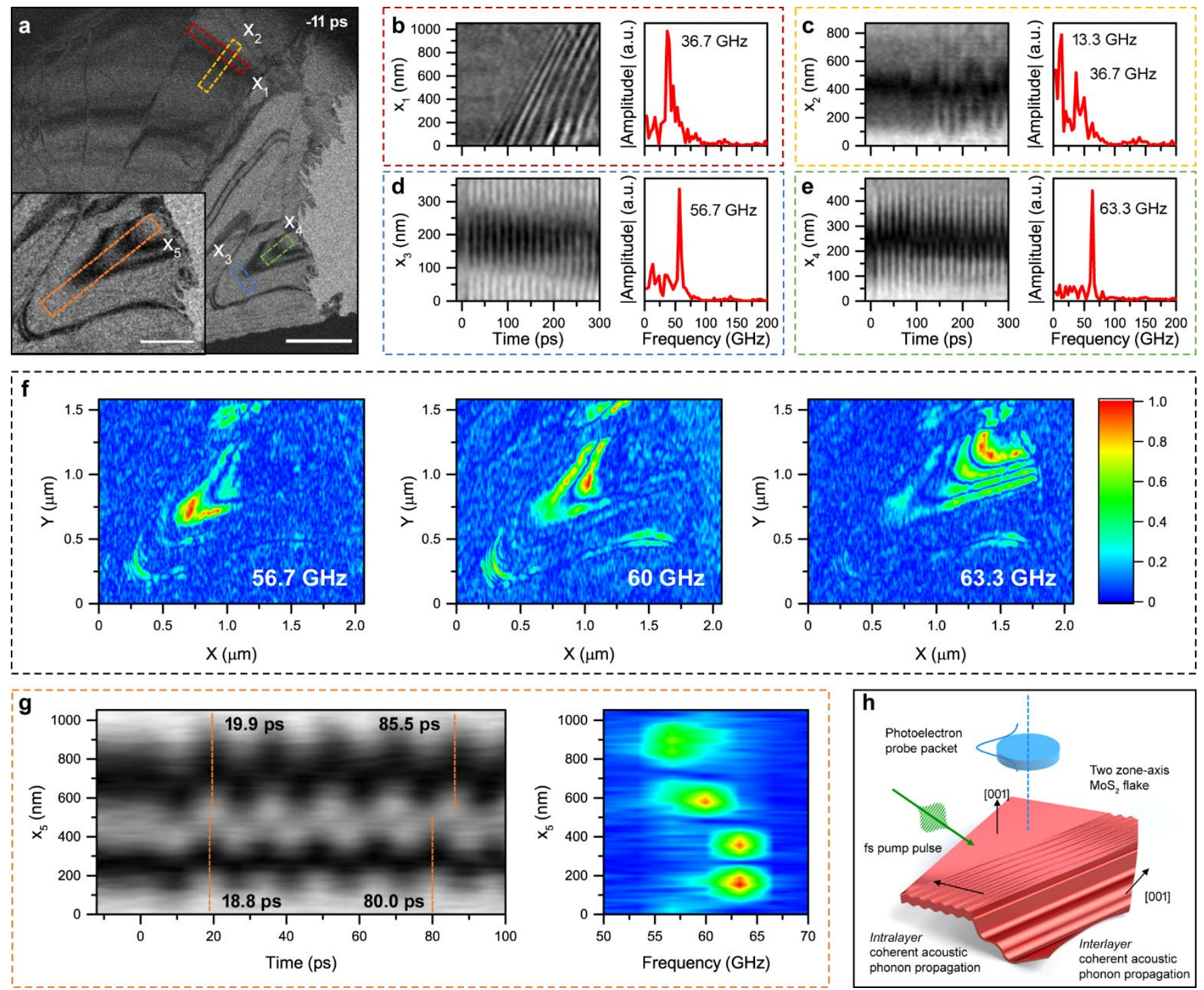

Figure 1. Localized anisotropic coherent acoustic-phonon dynamics and discrete, nanoscale few-layer dephasing. (a) Photoelectron bright-field image of the $\mathrm{MoS}_{2}$ specimen prior to in situ laser excitation. The dashed colored rectangles represent the regions of interest (ROIs) on which the subsequent spacetime and frequency-domain analyses were performed. The ROI labels $\left(x_{1}, x_{2}\right.$, etc. $)$ next to the colored dashed rectangles mark the zero spatial position in the space-time contour plots (STCPs) in panels (b)(e). Scale bar $=1 \mu \mathrm{m}$. Inset: Magnified view of the ROI analyzed in panel (f). Scale bar $=500 \mathrm{~nm}$. (b)-(e) STCPs generated from the ROIs highlighted in panel (a) along with the associated fast Fourier transform (FFT) of each intensity profile within the STCP between 0 and 300 ps. Prominent frequencies within each FFT are labeled. (f) Spatial frequency maps of the ROI in the panel-(a) inset showing normalized vibrational intensities (color bar) at 56.7, 60, and $63.3 \mathrm{GHz}$ (left, middle, and right, respectively). (g) Left: STCP generated from ROI $x_{5}$ highlighted in panel (a). The first and the fifth peaks of both bend-contour oscillations are labeled with the time at which they occur relative to experimental time zero. Right: Corresponding spatial FFT generated from the STCP illustrating the rapid dephasing and loss of coherence between the two nanoscale regions. (h) Illustration of the experimental concept and the observed phonon dynamics in the freestanding $\mathrm{MoS}_{2}$ flake. 